\title{
Synthesis, Spectral and Antimicrobial Studies of Some $O$-Vanillin-2-aminopyridine Schiff Base Complexes of Organyltellurium(IV)
}

\author{
ANJU MALIK, GOBIND GOYAT, VIKAS, K.K.VERMA and SAPANA GARG* \\ Department of Chemistry, Maharshi Dayanand University, Rohtak-124001, India \\ sapanagarg1511@gmail.com
}

Received 14 November 2017 / Accepted 28 November 2017

\begin{abstract}
The reactions of tellurium(IV) derivatives, $\mathrm{TeCl}_{4}, \mathrm{RTeCl}_{3}$ and $\mathrm{R}_{2} \mathrm{TeCl}_{2}$ with monobasic $O N N$ tridentate Schiff base ligand derived by the condensation of $o$-vanillin with 2-aminopyridine have been carried out to give tellurium(IV) complexes, $\left[\mathrm{TeCl}_{3}(2-\mathrm{APY}-\{o-\operatorname{Van}\})\right],\left[\mathrm{RTeCl}_{2}(2-\mathrm{APY}-\{\mathrm{o}-\mathrm{Van}\})\right]$ and $\left[\mathrm{R}_{2} \mathrm{TeCl}(2-\mathrm{APY}-\{o\right.$-Van $\left.\})\right]$ : where $\mathrm{R}=p$-methoxyphenyl, $p$-hydroxyphenyl, 3-methyl-4-hydroxyphcenyl. The synthesized Schiff Base (2-APY- $\{o-\mathrm{VanH}\})$ and their complexes were characterized by elemental analyses, conductivity measurements, FT-IR and proton NMR spectral studies. The Schiff base and some of their tellurium(IV) complexes display antifungal and antibacterial activities.
\end{abstract}

Keywords: Tridentate Schiff base, $o$-Vanillin, Organyltellurium, Antifungal, Antibacterial activities

\section{Introduction}

Schiff base derived from aromatic aldehydes have arisen the researchers interest because of its varied use in biological systems as antitubercular agent ${ }^{1,2}$, antibacterial ${ }^{3,4-9}$, antifungal ${ }^{6-10}$ and antitumor activities ${ }^{11,12}$. Schiff bases are known to be medicinally important and are used to design medicinal compounds ${ }^{13}$. Some Schiff bases bearing aryl groups or heterocyclic residues possess excellent biological activities have attracted the attention of many researchers in recent years ${ }^{14,15}$. The Schiff bases derived from $o$-vanillin and 2,3-diaminopyridine have been used as ionophores in a $\mathrm{Cu}(\mathrm{II})$ selective electrochemical sensors ${ }^{16}$.

Organyltellurium(IV) chlorides are known ${ }^{17-41}$ to behave as Lewis acids and form complexes with several N-, O- and S- donor bases. In view of this, we have investigated the reactions of tellurium(IV) chloride and organyltellurium(IV) chlorides with $o$-vanillin-2aminopyridine Schiff base to synthesize some new complexes of tellurium(IV).

\section{Experimental}

All chemicals used were of analytical reagent grade. All preparations were carried out under an atmosphere of dry $\mathrm{N}_{2}$ atmosphere. The solvents were purified by standard method ${ }^{42,43}$ 
before use. The purity of compounds was checked by TLC using Silica gel-G (Merck). Melting points were determined in open capillary tube and are uncorrected.

Carbon, hydrogen and nitrogen analyses were obtained microanalytically from SAIF, Panjab University Chandigarh on a ThermoFinnigan CHNS analyser. Conductivity was measured in DMSO at $25 \pm 2{ }^{\circ} \mathrm{C}$ with a dip type conductivity cell on a microprocessor based conductivity bridge type MICROSIL.

Infrared spectra $\left(4000-40 \mathrm{~cm}^{-1}\right)$ were recorded in $\mathrm{KBr}$ and Polyethylene pellets for MidIR and Far-IR respectively, on a FT-Infra-Red Spectrometer Model Nicolet IS50 (Thermo scientific). Proton NMR spectra were recorded in DMSO- $\mathrm{d}_{6}$ using tetramethylsilane as an internal reference on BRUKER AVANCE II 400 NMR spectrometer from CIL, Guru Jambeshwar University of Science and Technology, Hissar, Haryana, India.

\section{Preparation of organyltellurium(IV) trichlorides and diorganyltellurium(IV)}

\section{dichlorides}

4-Methoxyphenyltellurium(IV) trichloride ${ }^{44,45}$, bis( $p$-methoxyphenyl)tellurium(IV)dichloride ${ }^{45,46}$, 4-hydroxyphenyltellurium(IV)trichloride ${ }^{47}, \quad$ bis( $p$-hydroxyphenyl)tellurium(IV)dichloride ${ }^{47}$, 3-methyl-4-hydroxyphenyltellurium(IV)trichloride ${ }^{48}$ and bis(3-methyl-4-hydroxyphenyl)tellurium(IV)dichloride ${ }^{48}$ were prepared by the reactions of tellurium tetrachloride (Aldrich) with corresponding arenes i.e. anisole, phenol, $o$-cresol respectively, by the methods reported in the literature ${ }^{44-48}$.

\section{Preparation of Schiff base (2-APY-\{o-VanH\})}

The Schiff base was prepared by mixing equimolecular quantity of $o$-vanillin $(0.08$ mole, $12.17 \mathrm{~g})$ and 2-aminopyridine $(0.08$ mole, $7.52 \mathrm{~g})$ in $25 \mathrm{~mL}$ methanol in a round bottom flask equipped with a condenser ${ }^{49}$. The reaction mixture was refluxed on water bath for 4 hours. After cooling, the precipitated Schiff base was collected by filtration and recrystallized from methanol. The dark orange crystalline product was dried in a desiccator over anhydrous $\mathrm{CaCl}_{2}$ and kept over $\mathrm{P}_{4} \mathrm{O}_{10}$. Yield $=93 \%$, m.pt. $($ decomp. $)=82-84{ }^{\circ} \mathrm{C}$.

\section{Preparation of complexes}

Tellurium tetrachloride, organyltellurium(IV) trichlorides and diorganyltellurium(IV) dichlorides, when reacted with Schiff base (2-APY- $\{o-\mathrm{VanH}\})$ form solid complexes as described below.

\section{$\left[\mathrm{TeCl}_{3}(2-\mathrm{APY}-\{\mathrm{o}-\mathrm{Van}\})\right],\left[R T e C l_{2}(2-A P Y-\{o-\operatorname{Van}\})\right]$ and $\left[\mathrm{R}_{2} \mathrm{TeCl}(2-\mathrm{APY}-\{o-\operatorname{Van}\})\right]$}

The solid complexes were prepared by addition of $5 \mathrm{mmol}$ tellurium(IV) derivatives in about $25 \mathrm{~mL}$ anhydrous methanol to a hot solution of $5 \mathrm{mmol}$ Schiff base (2-APY- $\{o-\mathrm{VanH}\})$ in about $25 \mathrm{~mL}$ methanol with continuous stirring. The reaction mixture was refluxed on steam bath for 4 hours. The excess solvent was distilled off to obtain the desired products which were recrystallized from dry methanol. The coloured complexes crystallized out, which were filtered, washed with dry methanol and dried in a vacuum desiccator over $\mathrm{P}_{4} \mathrm{O}_{10}$.

\section{Results and Discussion}

Anisole ${ }^{44-46}$, phenol ${ }^{47}$ and $o-\mathrm{cresol}^{48}(\mathrm{R}-\mathrm{H})$ undergo Friedel Craft type condensation reaction when reacted with tellurium tetrachloride in boiling organic solvents to form organyltellurium(IV)trichlorides and diorganyltellurium(IV)dichlorides. This reaction involves the electrophilic substitution of the aromatic ring by a trichlorotellurium group at a position para to the methoxy / hydroxyl groups. 


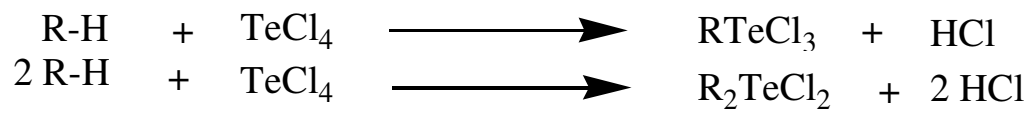

Preparation of Schiff base (2-APY- $\{o-\mathrm{VanH}\})$, by the reaction of $o$-vanillin with 2-aminopyridine can be represented by following equation.<smiles>COc1cccc(C=O)c1O</smiles>

$o$-Vanillin<smiles>Nc1ccccn1</smiles>

2-aminopyridine<smiles>COc1cccc(/C=N/c2ccccn2)c1O</smiles>

(2-APY- $\{o-\mathrm{VanH}\})$

Schiff base reacts with tellurium(IV)chloride, organyltellurium(IV)trichlorides and diorganyltellurium(IV)dichlorides to yield the coloured tellurium(IV) complexes.

$$
\begin{aligned}
\mathrm{TeCl}_{4}+(2-\mathrm{APY}-\{\mathrm{oVanH}\}) & \longrightarrow \mathrm{TeCl}_{3}(2-\mathrm{APY}-\{\mathrm{o}-\mathrm{Van}\}) \\
\mathrm{RTeCl}_{3}+(2-\mathrm{APY}-\{\mathrm{oVanH}\}) & \longrightarrow \mathrm{RTeCl}_{2}(2-\mathrm{APY}-\{\mathrm{oVanH}\}) \\
\mathrm{R}_{2} \mathrm{TeCl}_{2}+(2-\mathrm{APY}-\{\mathrm{oVanH}\}) & \longrightarrow \mathrm{R}_{2} \mathrm{TeCl}(2-\mathrm{APY}-\{\mathrm{oVanH}\})
\end{aligned}
$$

All the tellurium(IV) complexes are colored, crystalline solids, stable at room temperature and non-hygroscopic in nature. They are insoluble in non polar and less polar organic solvents, but are soluble in polar donor solvents like DMF, DMSO etc. The analytical data along with their physical properties are presented in Table 1.

\section{Conductance studies}

Molar conductance $\left(\Lambda_{\mathrm{M}}\right)$ data for organyltellurium(IV) Schiff base complexes in DMSO are compiled in Table 1. The $\Lambda_{\mathrm{M}}$ values at $c a \cdot 10^{-3} \mathrm{M}$ of complexes lies in the range 8.53-40.56 $\mathrm{S} \mathrm{cm} \mathrm{mol}^{-1}$ which predict the non-electrolyte to $1: 1$ weak electrolyte type behavior ${ }^{50,51}$ of these Schiff base complexes in DMSO, probably due to ionization into $\mathrm{TeCl}_{2}(2-\mathrm{APY}-\{o-$ $\operatorname{Van}\})^{+} / \mathrm{RTeCl}(2-\mathrm{APY}-\{o-\operatorname{Van}\})^{+} / \mathrm{R}_{2} \mathrm{Te}(2-\mathrm{APY}-\{o-\operatorname{Van}\})^{+}$and $\mathrm{Cl}^{-}$in DMSO. The higher $\Lambda_{\mathrm{M}}$ values for some complexes may be due to steric factors and donor behavior of DMSO to result in probable dissociation into solvated cation and 2-APY- $\{o-V a n\}^{-}$along with $\mathrm{Cl}^{-}$in DMSO. This conductance behavior of tellurium(IV) Schiff base complexes is different from those of transition metal complexes ${ }^{52}$ which are reported to be non-electrolytes.

\section{Infrared spectra}

Characteristics FT-IR bands of the free ligand and its complexes with their assignment are presented in Table 2. The FT-IR spectrum of the ligand ${ }^{10,49,53-55}$ showed a $v_{(\mathrm{C}=\mathrm{N})}$ peak at $1610 \mathrm{~cm}^{-1}, v_{(\mathrm{C}-\mathrm{N}-\mathrm{C})}$ peak at $1462 \mathrm{~cm}^{-1}$ and the absence of $v_{(\mathrm{C}=\mathrm{O})}$ at $1643 \mathrm{~cm}^{-1}$ and $v_{\left(\mathrm{NH}_{2}\right)}$ peaks around $3250-3300 \mathrm{~cm}^{-1}$ is because of Schiff base condensation.

The spectrum also showed a broad band at $\approx 3064 \mathrm{~cm}^{-1}$ due to $v_{(\mathrm{OH})}$. The strong band at $1610 \mathrm{~cm}^{-1}$ in the IR spectrum of free ligand assigned to $v_{(\mathrm{C}=\mathrm{N})}$ that changes in the FT-IR spectra of the complexes ${ }^{6,49,53-55}$ (towards lower frequency region), indicating coordination through azomethine nitrogen ${ }^{56,57}$.

The $v_{(\mathrm{C}-\mathrm{N}-\mathrm{C})}$ strong band at $1462 \mathrm{~cm}^{-1}$ in the free ligand shifts to higher frequency upon complexation due to coordination with nitrogen atom of pyridine ring. The broad band appearing around $3064 \mathrm{~cm}^{-1}$ in free ligand assignable to $v_{(\mathrm{OH})}$ vibration mode is absent in the spectra of complexes suggesting the coordination through oxygen of phenolic group to the tellurium centre. 
Table 1. Analytical data, molar conductance and physical properties for Schiff base (2-APY-\{o-VanH $\})$ complexes of tellurium(IV) values of $\Lambda_{\mathrm{M}}$ reported $^{50,51}$ for 1:1 electrolytes in DMSO $=50-70 \mathrm{~S} \mathrm{~cm}^{2} \mathrm{~mol}^{-1}$

\begin{tabular}{|c|c|c|c|c|c|c|c|c|c|c|}
\hline \multirow{2}{*}{ 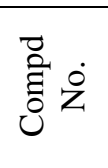 } & \multirow[b]{2}{*}{$\begin{array}{l}\text { Complex } \\
\text { (R) }\end{array}$} & \multirow[b]{2}{*}{$\begin{array}{l}\text { Empirical formula } \\
\text { (Formula Wt.) }\end{array}$} & \multirow[b]{2}{*}{$\begin{array}{l}\text { Colour, } \\
\text { yield,\% }\end{array}$} & \multirow{2}{*}{$\begin{array}{l}\text { M. Pt. } \\
{ }^{\circ} \mathrm{C} \\
\text { dec. }\end{array}$} & \multicolumn{5}{|c|}{ Analyses \% Found (Calculated) } & \multirow{2}{*}{$\begin{array}{c}\Lambda_{\mathrm{M}} \text { at } c a . \\
10^{-3} \mathrm{M} \\
\mathrm{S} \mathrm{cm}^{2} \mathrm{~mol}^{-1} \\
\text { in DMSO }\end{array}$} \\
\hline & & & & & $\mathrm{C}$ & $\mathrm{H}$ & $\mathrm{N}$ & $\mathrm{Te}$ & $\mathrm{Cl}$ & \\
\hline $\begin{array}{l}\text { Schiff } \\
\text { Base }\end{array}$ & $(2-\mathrm{APY}-\{o-\operatorname{VanH}\})$ & $\begin{array}{c}\mathrm{C}_{13} \mathrm{H}_{12} \mathrm{~N}_{2} \mathrm{O}_{2} \\
(228.15)\end{array}$ & $\begin{array}{c}\text { Dark } \\
\text { orange (93) }\end{array}$ & $82-84$ & $\begin{array}{l}68.28 \\
(68.43)\end{array}$ & $\begin{array}{c}5.43 \\
(5.26)\end{array}$ & $\begin{array}{c}12.14 \\
(12.28)\end{array}$ & - & - & - \\
\hline 1 & $\begin{array}{c}\mathrm{TeCl}_{3}(2-\mathrm{APY}- \\
\{o-\operatorname{Van}\})\end{array}$ & $\begin{array}{c}\mathrm{C}_{13} \mathrm{H}_{11} \mathrm{Cl}_{3} \mathrm{~N}_{2} \mathrm{O}_{2} \mathrm{Te} \\
(461.24)\end{array}$ & $\begin{array}{l}\text { Light } \\
\text { brown (78) }\end{array}$ & 193-195 & $\begin{array}{l}33.61 \\
(33.85)\end{array}$ & $\begin{array}{c}2.47 \\
(2.38)\end{array}$ & $\begin{array}{c}5.96 \\
(6.07)\end{array}$ & $\begin{array}{c}27.54 \\
(27.66)\end{array}$ & $\begin{array}{l}22.95 \\
(23.09)\end{array}$ & 8.53 \\
\hline 2 & $\begin{array}{c}\mathrm{RTeCl}_{2}(2-\mathrm{APY}- \\
\{o \text {-Van }\}) \\
\text { (4-methoxyphenyl) }\end{array}$ & $\begin{array}{c}\mathrm{C}_{20} \mathrm{H}_{18} \mathrm{Cl}_{2} \mathrm{~N}_{2} \mathrm{O}_{3} \mathrm{Te} \\
(532.81)\end{array}$ & $\begin{array}{l}\text { Yellowish } \\
\text { green (76) }\end{array}$ & $174-176$ & $\begin{array}{c}44.89 \\
(45.08)\end{array}$ & $\begin{array}{c}3.52 \\
(3.38)\end{array}$ & $\begin{array}{l}5.15 \\
(5.26)\end{array}$ & $\begin{array}{c}23.84 \\
(23.95)\end{array}$ & $\begin{array}{c}13.02 \\
(13.14)\end{array}$ & 15.92 \\
\hline 3 & $\begin{array}{c}\mathrm{RTeCl}_{2}(2-\mathrm{APY}- \\
\{o \text {-Van }\}) \\
\text { (4-hydroxyphenyl) }\end{array}$ & $\begin{array}{c}\mathrm{C}_{19} \mathrm{H}_{16} \mathrm{Cl}_{2} \mathrm{~N}_{2} \mathrm{O}_{3} \mathrm{Te} \\
(518.80)\end{array}$ & $\begin{array}{c}\text { Dark } \\
\text { brown (82) }\end{array}$ & $203-205$ & $\begin{array}{l}43.75 \\
(43.98)\end{array}$ & $\begin{array}{c}3.20 \\
(3.08)\end{array}$ & $\begin{array}{c}5.23 \\
(5.40)\end{array}$ & $\begin{array}{l}24.47 \\
(24.59)\end{array}$ & $\begin{array}{c}13.52 \\
(13.69)\end{array}$ & 19.08 \\
\hline 4 & $\begin{array}{c}\mathrm{RTeCl}_{2}(2-\mathrm{APY}- \\
\{o-\mathrm{Van}\}) \\
\text { (3-methyl-4- } \\
\text { hydroxyphenyl) }\end{array}$ & $\begin{array}{c}\mathrm{C}_{20} \mathrm{H}_{18} \mathrm{Cl}_{2} \mathrm{~N}_{2} \mathrm{O}_{3} \mathrm{Te} \\
(532.81)\end{array}$ & $\begin{array}{l}\text { Brick } \\
\text { red (77) }\end{array}$ & $181-183$ & $\begin{array}{c}44.92 \\
(45.08)\end{array}$ & $\begin{array}{c}3.24 \\
(3.38)\end{array}$ & $\begin{array}{c}5.18 \\
(5.26)\end{array}$ & $\begin{array}{l}23.82 \\
(23.95)\end{array}$ & $\begin{array}{l}13.04 \\
(13.14)\end{array}$ & 16.33 \\
\hline 5 & $\begin{array}{c}\mathrm{R}_{2} \mathrm{TeCl}(2-\mathrm{APY}- \\
\{o \text {-Van }\}) \\
\text { (4-methoxyphenyl) }\end{array}$ & $\begin{array}{c}\mathrm{C}_{27} \mathrm{H}_{25} \mathrm{ClN}_{2} \mathrm{O}_{4} \mathrm{Te} \\
(604.38)\end{array}$ & $\begin{array}{l}\text { Light } \\
\text { brown (75) }\end{array}$ & 197-199 & $\begin{array}{c}53.52 \\
(53.65)\end{array}$ & $\begin{array}{c}4.03 \\
(4.14)\end{array}$ & $\begin{array}{c}4.52 \\
(4.64)\end{array}$ & $\begin{array}{l}21.02 \\
(21.11)\end{array}$ & $\begin{array}{c}5.75 \\
(5.87)\end{array}$ & 24.67 \\
\hline 6 & $\begin{array}{c}\mathrm{R}_{2} \mathrm{TeCl}(2-\mathrm{APY}- \\
\{o \text {-Van }\}) \\
\text { (4-hydroxyphenyl) }\end{array}$ & $\begin{array}{c}\mathrm{C}_{25} \mathrm{H}_{21} \mathrm{ClN}_{2} \mathrm{O}_{4} \mathrm{Te} \\
(576.36)\end{array}$ & $\begin{array}{l}\text { Yellowish } \\
\text { brown (85) }\end{array}$ & $200-202$ & $\begin{array}{c}51.94 \\
(52.09)\end{array}$ & $\begin{array}{c}3.74 \\
(3.64)\end{array}$ & $\begin{array}{c}4.72 \\
(4.86)\end{array}$ & $\begin{array}{l}22.04 \\
(22.14)\end{array}$ & $\begin{array}{c}6.05 \\
(6.16)\end{array}$ & 40.56 \\
\hline 7 & $\begin{array}{c}\mathrm{R}_{2} \mathrm{TeCl}(2-\mathrm{APY}- \\
\{o \text {-Van }\}) \\
\text { (3-methyl-4- } \\
\text { hydroxyphenyl) }\end{array}$ & $\begin{array}{c}\mathrm{C}_{27} \mathrm{H}_{25} \mathrm{ClN}_{2} \mathrm{O}_{4} \mathrm{Te} \\
(604.38)\end{array}$ & $\begin{array}{c}\text { Dark } \\
\text { brown (73) }\end{array}$ & $177-179$ & $\begin{array}{c}53.47 \\
(53.65)\end{array}$ & $\begin{array}{c}4.25 \\
(4.14)\end{array}$ & $\begin{array}{c}4.52 \\
(4.64)\end{array}$ & $\begin{array}{c}20.97 \\
(21.11)\end{array}$ & $\begin{array}{c}5.73 \\
(5.87)\end{array}$ & 39.93 \\
\hline
\end{tabular}


In the spectra of complexes, the bands observed in the 289- $290 \mathrm{~cm}^{-1}$ and $418-419 \mathrm{~cm}^{-1}$ region might be due to $v_{(\mathrm{Te}-\mathrm{O})}{ }^{54,55,58-60}$ and $\mathrm{v}_{(\mathrm{Te}-\mathrm{N})}{ }^{60}$ further support the involvement of phenolic oxygen, azomethine and pyridine nitrogen atoms of Schiff base in the coordination. Further, presence of aryl groups of Rte and $\mathrm{R}_{2} \mathrm{Te}$ may result in mixing of certain bands, thus making independent assignments very difficult. Thus, the Schiff base ligand is coordinated to the tellurium atom as uninegative $O N N$ tridentate ligand give rise to two chelate (six and four membered) with ring tellurium centre.

Table 2. Important IR data $\left(\mathrm{cm}^{-1}\right)$ of the Schiff base (2-APY- $\left.\{0-\mathrm{VanH}\}\right)$ and complexes

\begin{tabular}{cccccc}
\hline $\begin{array}{c}\text { Compound } \\
\text { No. }\end{array}$ & $\begin{array}{c}\text { (Phenolic) } \\
v_{(\mathrm{OH})}\end{array}$ & $\begin{array}{c}\text { (Azomethine) } \\
\mathrm{v}_{(\mathrm{C}=\mathrm{N})}\end{array}$ & $\begin{array}{c}\text { (Pyridine) } \\
v_{(\mathrm{C}-\mathrm{N}-\mathrm{C})}\end{array}$ & $v_{(\mathrm{Te}-\mathrm{O})}$ & $v_{(\mathrm{Te}-\mathrm{N})}$ \\
\hline $\begin{array}{c}(2-\mathrm{APY}- \\
\{o \text {-VanH }\})\end{array}$ & $3064 \mathrm{~b}$ & $1610 \mathrm{~s}$ & $1462 \mathrm{~s}$ & - & - \\
1 & - & $1586 \mathrm{sh}$ & $1488 \mathrm{~s}$ & $289 \mathrm{~s}$ & $419 \mathrm{~s}$ \\
2 & - & $1577 \mathrm{~s}$ & $1489 \mathrm{~s}$ & $289 \mathrm{~s}$ & $419 \mathrm{~s}$ \\
3 & - & $1596 \mathrm{mb}$ & $1487 \mathrm{mb}$ & $289 \mathrm{~s}$ & $418 \mathrm{~s}$ \\
4 & - & $1576 \mathrm{~s}$ & $1488 \mathrm{~m}$ & $289 \mathrm{~s}$ & $419 \mathrm{~s}$ \\
5 & - & $1590 \mathrm{~s}$ & $1489 \mathrm{mb}$ & $289 \mathrm{~s}$ & $419 \mathrm{~s}$ \\
6 & - & $1592 \mathrm{~s}$ & $1487 \mathrm{~m}$ & $290 \mathrm{~s}$ & $419 \mathrm{~s}$ \\
7 & - & $1588 \mathrm{~s}$ & $1489 \mathrm{~s}$ & $290 \mathrm{~s}$ & $419 \mathrm{~s}$ \\
\hline
\end{tabular}

( $s=$ strong, $m=$ medium, $b=$ broad, $s h=$ shoulder $)$

\section{${ }^{1} H$ NMR spectra}

DMSO- $\mathrm{d}_{6}$ was used as a solvent to measure the ${ }^{1} \mathrm{H}$ NMR spectra of free ligand as well as its complexes and data is presented in Table 3.

Table 3. ${ }^{1} \mathrm{H}$ NMR spectral data of Schiff base (2-APY- $\left.\{o-\mathrm{VanH}\}\right)$ and complexes

\begin{tabular}{|c|c|c|c|c|c|}
\hline $\begin{array}{l}\text { Compound } \\
\text { Number }\end{array}$ & $\begin{array}{c}\text { (Phenolic) } \\
-\mathrm{OH} \\
\delta \mathrm{ppm}\end{array}$ & $\begin{array}{c}\text { (Azomethine) } \\
-\mathbf{H C}=\mathrm{N} \\
\delta \mathrm{ppm}\end{array}$ & $\begin{array}{l}\text { (Ar rings } \\
\text { protons) } \\
\delta \mathrm{ppm}\end{array}$ & $\begin{array}{c}-\mathrm{CH}_{3} / \\
-\mathrm{OCH}_{3}^{*} \\
\delta \mathrm{ppm}\end{array}$ & $\begin{array}{c}-\mathrm{OH} \text { of } \\
\text { Rte/R }{ }_{2} \mathrm{Te} \\
\delta \mathrm{ppm}\end{array}$ \\
\hline $\begin{array}{c}(2-\mathrm{APY}- \\
\{\mathrm{o}-\mathrm{VanH}\})\end{array}$ & $13.935(\mathrm{~s}, 1 \mathrm{H})$ & $9.446(\mathrm{~s}, 1 \mathrm{H})$ & $\begin{array}{c}6.864-8.519 \\
(\mathrm{~cm}, 7 \mathrm{H})\end{array}$ & $3.944\left(\mathrm{~s}, 3 \mathrm{H}^{*}\right)$ & - \\
\hline 1 & - & $10.223(\mathrm{~s}, 1 \mathrm{H})$ & $\begin{array}{c}6.859-7.941 \\
(\mathrm{~cm}, 7 \mathrm{H})\end{array}$ & $3.996\left(\mathrm{~s}, 3 \mathrm{H}^{*}\right)$ & - \\
\hline 2 & - & $10.234(\mathrm{~s}, 1 \mathrm{H})$ & $\begin{array}{c}6.848-8.330 \\
(\mathrm{~cm}, 11 \mathrm{H})\end{array}$ & $3.810\left(\mathrm{~s}, 6 \mathrm{H}^{*}\right)$ & - \\
\hline 3 & - & $10.234(\mathrm{~s}, 1 \mathrm{H})$ & $\begin{array}{c}6.844-8.101 \\
(\mathrm{~cm}, 11 \mathrm{H})\end{array}$ & $3.420\left(\mathrm{~s}, 3 \mathrm{H}^{*}\right)$ & $\begin{array}{l}10.020 \\
(\mathrm{~s}, 1 \mathrm{H})\end{array}$ \\
\hline 4 & - & $10.270(\mathrm{~s}, 1 \mathrm{H})$ & $\begin{array}{c}6.842-8.240 \\
(\mathrm{~cm}, 10 \mathrm{H})\end{array}$ & $\begin{array}{l}2.509(\mathrm{~s}, 3 \mathrm{H}) / \\
3.841\left(\mathrm{~s}, 3 \mathrm{H}^{*}\right)\end{array}$ & $\begin{array}{l}9.101 \\
(\mathrm{~s}, 1 \mathrm{H})\end{array}$ \\
\hline 5 & - & $10.234(\mathrm{~s}, 1 \mathrm{H})$ & $\begin{array}{c}6.849-7.932 \\
(\mathrm{~cm}, 15 \mathrm{H})\end{array}$ & $3.372\left(\mathrm{~s}, 9 \mathrm{H}^{*}\right)$ & - \\
\hline 7 & - & $10.276(\mathrm{~s}, 1 \mathrm{H})$ & $\begin{array}{c}6.832-8.099 \\
(\mathrm{~cm}, 13 \mathrm{H})\end{array}$ & $\begin{array}{l}2.508(\mathrm{~s}, 6 \mathrm{H}) / \\
3.388\left(\mathrm{~s}, 3 \mathrm{H}^{*}\right)\end{array}$ & $\begin{array}{l}10.009 \\
(\mathrm{~s}, 2 \mathrm{H})\end{array}$ \\
\hline
\end{tabular}

$s=$ singlet, $\mathrm{cm}=$ complex multiplet, Spectra of compound number 6 not well resolved due to poor solubility 
The ${ }^{1} \mathrm{H}$ NMR spectrum of the ligand showed signal at $\delta 9.446 \mathrm{ppm}$ corresponding to azomethine proton. The singlet observed at about $\delta 13.935 \mathrm{ppm}$ due to phenolic proton of the ligand. The complex multiplets corresponding aromatic protons appeared between $\delta$ 6.864-8.519 ppm. The hydroxyl proton (phenolic -OH) disappears in the tellurium(IV) complexes indicating that the -OH group has been deprotonated and bonded to metal centre.

Another signal displays a downfield shift from 9.446 to $10.223-10.276 \delta \mathrm{ppm}$, suggest the decrease of electron density and deshielding of azomethine proton, as a result of which nitrogen atom of azomethine group coordination to the tellurium centre $30,31,49,53,61$. Independent assignments to the aryl protons of Schiff base and $\mathrm{RTe} / \mathrm{R}_{2} \mathrm{Te}$ are not possible due to overlapping of signals in this region.

On the basis of spectral studies, it may be concluded that Schiff base acts as uninegative $(O N N)$ tridentate ligand, resulting in the formation of hexacoordinated tellurium(IV) complexes probably in a distorted octahedral geometry and proposed structures are shown in Figure 1.<smiles>COc1cccc(/C=N/c2ccccn2)c1O</smiles>

(2-APY- $\{o-\mathrm{VanH}\})$<smiles>[R][Te]1(Cl)(Cl)c2cccc(OC)c2Oc2cnn1c1cccc2-1</smiles>

$\mathrm{RTeCl}_{2}(2-\mathrm{APY}-\{o-\mathrm{Van}\})$<smiles></smiles>

$\mathrm{TeCl}_{3}(2-\mathrm{APY}-\{o-\mathrm{Van}\})$<smiles>[R][Te]1([R])Oc2c(cccc2OC)/C=N\c2cccn21</smiles>

$\mathrm{R}_{2} \mathrm{TeCl}(2-\mathrm{APY}-\{o$-Van $\})$

Figure 1. Proposed structures of Schiff base (2-APY-\{o-VanH $\}$ ) and tellurium(IV) complexes

\section{Biological studies}

The $o$-vanillin-2-aminopyridine Schiff base (2-APY-\{o-VanH $\}$ ) and newly synthesized organyltellurium(IV) Schiff base complexes were evaluated for their antimicrobial activities in vitro against Gram-positive bacteria (S. aureus MTCC 96 and S. pyogenes MTCC 442), Gram-negative bacteria ( $P$. aeruginosa MTCC 1688 and E. coli MTCC 443) and fungi C. albicans MTCC 227, A. niger MTCC 282 and A. clavatus MTCC 1323. The evaluation of the biological activities was carried by "Broth Dilution Method". After incubation the inhibition was measured and the activity of complexes was compared with standard drugs ampicillin and chloramphenicol for antibacterial, nystatin and greseofulvin for antifungal activities $^{62}$. The data is given in Table 4 .

The data show the complexes of organyltellurium(IV) exhibited more antibacterial activity than antifungal activity as compared to free ligand. The complex no. 3 i.e. $\left[\mathrm{RTeCl}_{2}(2\right.$-APY- $\{o$-Van $\left.\})\right]$ : where $\mathrm{R}=4$-hydroxyphenyl, show substantial activity against two bacterial strains ( $S$. aureus and S. pyogenes) than Schiff base itself as well as other complexes and even comparable to standard drug ampicillin and complex no. 1 i.e. $\left[\mathrm{TeCl}_{3}\right.$ 
(2-APY-\{o-Van $\})$ showed good antibacterial activity against two bacterial strains (P. aeruginosa and E. coli). Complex no. 2 and 6 i.e. $\left[\mathrm{RTeCl}_{2}(2-\mathrm{APY}-\{o-\mathrm{Van}\})\right]$ : where $\mathrm{R}=$ 4-methoxyphenyl and $\left[\mathrm{R}_{2} \mathrm{TeCl}(2-\mathrm{APY}-\{o\right.$-Van $\left.\})\right]$ : where $\mathrm{R}=4$-hydroxyphenyl respectively, showed moderate antibacterial activity against all four pathogenic bacterial strains.

Table 4. Minimum inhibitory concentration MIC $(\mu \mathrm{g} / \mathrm{mL})$ of Schiff base (2-APY- $\{o-\mathrm{VanH}\})$ and complexes

\begin{tabular}{|c|c|c|c|c|c|c|c|}
\hline \multirow[b]{2}{*}{ Compound No. } & \multicolumn{4}{|c|}{ Bacterial strain } & \multicolumn{3}{|c|}{ Fungal strain } \\
\hline & $\begin{array}{c}\text { S.aureus } \\
\text { MTCC } \\
96\end{array}$ & $\begin{array}{c}S . \\
\text { pyogenes } \\
\text { MTCC } \\
442\end{array}$ & $\begin{array}{c}P . \\
\text { aeruginosa } \\
\text { MTCC } 1688\end{array}$ & $\begin{array}{c}\text { E. } \\
\text { coli } \\
\text { MTCC } \\
443\end{array}$ & $\begin{array}{c}C . \\
\text { albicans } \\
\text { MTCC } \\
227\end{array}$ & $\begin{array}{c}\text { A.niger } \\
\text { MTCC } \\
282\end{array}$ & $\begin{array}{c}\text { A. } \\
\text { clavatus } \\
\text { MTCC } \\
1323\end{array}$ \\
\hline $\begin{array}{c}(2-\mathrm{APY}- \\
\{o-\mathrm{VanH}\})\end{array}$ & 500 & 500 & 250 & 250 & 500 & 1000 & 1000 \\
\hline 1 & 500 & 500 & 100 & 62.5 & 1000 & 500 & 1000 \\
\hline 2 & 250 & 250 & 200 & 200 & 1000 & 500 & 1000 \\
\hline 3 & 100 & 100 & 500 & 500 & 500 & 1000 & $>1000$ \\
\hline 6 & 250 & 250 & 200 & 250 & 1000 & $>1000$ & $>1000$ \\
\hline & Standa & d Drugs & & & & & \\
\hline Ampicillin & 250 & 100 & 100 & 100 & - & - & - \\
\hline Chloramphenicol & 50 & 50 & 50 & 50 & - & - & - \\
\hline Nystatin & - & - & - & - & 100 & 100 & 100 \\
\hline Greseofulvin & - & - & - & - & 500 & 100 & 100 \\
\hline
\end{tabular}

\section{Conclusion}

Schiff base (2-APY- $\{o-\mathrm{VanH}\})$ derived from $o$-vanillin with 2-aminopyridine have been synthesized. Schiff base when reacted with tellurium tetrachloride, organyltellurium(IV) trichlorides and diorganyltellurium(IV) dichlorides in 1:1 molar ratios yield $\left[\mathrm{TeCl}_{3}(2-\mathrm{APY}-\right.$ $\{\operatorname{Van}\})],\left[\mathrm{RTeCl}_{2}(2-\mathrm{APY}-\{\operatorname{Van}\})\right]$ and $\left[\mathrm{R}_{2} \mathrm{TeCl}(2-\mathrm{APY}-\{\operatorname{Van}\})\right]$ : where $\mathrm{R}=4$-methoxyphenyl, 4hydroxyphenyl, 3-methyl-4-hydroxyphenyl type complexes. The structure of the purified products was confirmed by FT-IR, ${ }^{1} \mathrm{H}$ NMR spectral studies which predict the hexacoordinated tellurium(IV) complexes probably in a distorted octahedral geometry by the monobasic tridentate $(O N N)$ Schiff base. Some of these complexes possess good antimicrobial activity.

\section{Acknowledgement}

The authors are grateful to M. D. University, Rohtak for providing the necessary facilities. One of the authors (AM) is also thankful to HSCST, Haryana for providing a fellowship. We also thank SAIF, Panjab University Chandigarh for providing the CHN analyses, ${ }^{1} \mathrm{H}$ NMR spectral data from CIL, Guru Jambeshwar University of Science and Technology, Hissar and the Microcare Laboratory, Surat, for providing antimicrobial activities.

\section{References}

1. Rajasekaran A, Periasamy M and Venkatesan S, J Dev Bio Tissue Eng., 2010, 2(1), 5-13.

2. $\quad$ Ilango K and Arun Kumar S, Trop J Pharm Res., 2011, 10(2), 227-232.

3. Lei Shi, Hui- Ming Ge, Shu Hua Tan, Huan Qui Li, Yong-Chun Song, Hai Liang Zhu and Ren Xiang Tan, Eur J Med Chem., 2007, 42(4), 558-564;

DOI:10.1016/j.ejmech.2006.11.010

4. Sridhar S K, Saravanan M and Ramesh A, Eur J Med Chem., 2001, 36(7-8), 615-625; DOI:10.1016/S0223-5234(01)01255-7 
5. Mladenova R, Ignatova M, Manolova N, Petrova T and Rashkov I, Eur Polym J., 2002, 38(5), 989-999; DOI:10.1016/S0014-3057(01)00260-9

6. Panneerselvem P, Nair R R, Vijayalakshmi G, Subramanian E H and Sridhar S K, Eur J Med Chem., 2005, 40(2), 225-229; DOI:10.1016/j.ejmech.2004.09.003

7. Walsh O M, Meegan M J, Prendergast R M and Nakib T A, Eur J Med Chem., 1996, 31(12), 989-1000; DOI:10.1016/S0223-5234(97)86178-8

8. Pandeya S N, Sriram D, Nath G and DeClercq E, Eur J Pharm Sci., 1999, 9(1), 25-31; DOI:10.1016/S0928-0987(99)00038-X

9. Pandeya S N, Sriram D, Nath G and DeClercq E, Pharm Acta Helv., 1999, 74(1), 11 17; DOI:10.1016/S0031-6865(99)00010-2

10. Ramesh R and Maheswaran S, J Inorg. Biochem., 2003, 96(4), 457-462; DOI:10.1016/S0162-0134(03)00237-X

11. Liu M C, Lin T S and Sartorelli A C, J Med Chem., 1992, 35(20), 3672-3677; DOI:10.1021/jm00098a012

12. Hodnett E M and Dunn J W, J Med Chem., 1970, 13(4), 768-770;

DOI:10.1021/jm00298a054

13. Capdeville R, Buchdunger E, Zimmermann $\mathrm{J}$ and Matter A, Nature Reviews Drug Discovery, 2002, 1(7), 493-502; DOI:10.1038/nrd839.

14. Halla B S, Rao B S, Shridhara K and Akberali P M, Farmaco, 2000, 55(5), 338-344; DOI:10.1016/S0014-827X(00)00033-1

15. Chaha V K, Ranwa N S and Dadheech P K, J Phytol Res., 1998, 11, 201-202.

16. Singh L P and Bhatnagar J M, Talanta., 2004, 64(2), 313-319;

DOI:10.1016/j.talanta.2004.02.020

17. Goyat G, Garg S and Verma K K, Chem Sci Trans., 2016, 5(2), 479-487; DOI:10.7598/cst2016.1204

18. Goyat G, Garg S and Verma K K, Res J Pharm Biol Chem Sci., 2016, 7(2), 869-877.

19. Goyat G, Malik A, Garg S and Verma K K, Int J Chem Sci., 2016, 14(3), 1498-1510.

20. Goyat G, Malik A, Garg S and Verma K K, J Chem Pharm Res., 2016, 8(4), 218-223.

21. Goyat G, Malik A, Garg S and Verma K K, Int J Chem Sci., 2016, 14(1), 387-398.

22. Goyat G, Malik A, Garg S and Verma K K, Der Pharma Chemica, 2016, 8(2), 198-203.

23. Wynne K J and Pearson P S, Inorg Chem., 1971, 10(12), 2735-2739;

DOI:10.1021/ic50106a022

24. Wynne K J and Pearson P S, J Chem Soc Commun., 1970, 556.

25. Wynne K J, Clark A J and Berg M, J Chem Soc Dalton., 1972, 2370-2374; DOI:10.1039/DT9720002370

26. Clark E R, Collet A J and Naik D G, J Chem Soc Dalton., 1973, 1961-1962; DOI:10.1039/DT9730001961

27. Berg M C, Diss Abstr Int., 1972, 33, 2982.

28. Srivastava T N, Singh M and Singh H B, Indian J Chem., 1982, 21A, 307.

29. Srivastava T N, Srivastava R C and Srivastava M, Indian J Chem., 1982, 21A, 539.

30. Srivastava T N, Srivastava R C and Srivastava V K, J Indian Chem Soc., 1983, 60, 891.

31. Garad M V, Polyhedron, 1985, 4(8), 1353-1355; DOI:10.1016/S02775387(00)86963-6

32. Verma K K and Reena K K, Synth React Inorg Met Org Chem., 1999, 29(3), 499-512; DOI:10.1080/00945719909349465

33. Verma K K, Dahiya R and Soni D, Synth React Inorg Met Org Chem., 1999, 29(6), 1033-1052; DOI:10.1080/00945719909349509 
34. Verma K K and Dahiya R, Synth React Inorg Met Org Chem., 1999, 29(7), 1299-1314; DOI:10.1080/00945719909349529

35. Verma K K and Reena, Phosphorus Sulfur Silicon Related Elements, 1999, 148(1), 227-234; DOI:10.1080/10426509908037013

36. Verma K K and Seema, Int J Chem Sci., 2008, 6, 371-380.

37. Malik A, Verma K K and Garg S, Res J Pharm Biol Chem Sci., 2017, 8(6), 190-198.

38. Malik A, Verma K K and Garg S, Chem Sci Trans., 2018, 7(2), 191-200; DOI:10.7598/cst2018.1464

39. Srivastava S, Soni D K and Gupta H S, J Indian Chem Soc., 1996, 73, 255.

40. Narwal J K, Chhabra S, Malik R K, Garg S and Verma K K, Oriental J Chem., 2013, 29, 1339-1349.

41. Chhabra S and Verma K K, J Chem Pharm Res., 2010, 2, 569-575.

42. Vogel A I, A Test Book of Organic Chemistry, $3^{\text {rd }}$ Edn., Longman: London, 1975.

43. Weissberger A, Ed., Technique of Organic Chemistry, Vol. 7, $2^{\text {nd }}$ Edn., Interscience Publishers, Inc.: N. Y., 1967.

44. Morgan G T and Kellet R E, J Chem Soc., 1926, 1080-1088;

DOI:10.1039/JR9262901080

45. Petragnani $\mathrm{N}$ and Stefani $\mathrm{H}$ A, Tellurium in Organic Chemistry, $2^{\text {nd }}$ Edn., Academic Press: London, 2007, 76.

46. Bergman J, Tetrahedron, 1972, 28(12), 3323-3331; DOI:10.1016/S0040-4020(01)93674-9

47. Khandelwal B L, Kumar K and Berry F J, Inorg Chim Acta, 1981, 47, 135-137; DOI:10.1016/S0020-1693(00)89319-6

48. Khandelwal B L, Kumar K and Raina K, Synth React Inorg Met Org Chem., 1981, 11(1), 65-78; DOI:10.1080/00945718108059276

49. Abdel-Latif S A, Hassib H B and Issa Y M, Spectrochim Acta(A), Mole Biomole Spectr., 2007, 67(3-4), 950-957; DOI:10.1016/j.saa.2006.09.013

50. Geary W J, Coord Chem Rev., 1971, 7(1), 81-122; DOI:10.1016/S0010-8545(00)80009-0

51. Greenwood N N, Straughan B P and Wilson A E, J Chem Soc A, 1968, 2209-2212; DOI:10.1039/J19680002209

52. Srivastava K P, Singh A and Singh S K, J Appl Chem., 2014, 7(4), 16-23.

53. Dharmaraj N, Trans Metal Chem., 2001, 26(1-2), 105-109;

DOI:10.1023/A:1007132408648

54. Verma K K, Soni D and Verma S, Phosphorus, Sulfur Silicon, 2000, 166(1), 231-241; DOI:10.1080/10426500008076544

55. Pant B C, Mc Whinnie W R and Dance N S, J Organmetal Chem., 1973, 63, 305-310; DOI;10.1016/S0022-328X(73)80043-9

56. Kohawole G A and Patel K S, J Chem Soc., Dalton Trans., 1981, 1241-1245; DOI:10.1039/DT9810001241

57. Percy G C and Stenton H S, Spectrochim Acta, 1976, 32(10), 1615-1621; DOI:10.1016/0584-8539(76)80263-2

58. Srivastava T N and Singh J D, Indian J Chem., 1987, 26A, 260.

59. Chauhan S, Garg S and Verma K K, Chem Sci Trans., 2016, 5(2), 431-441; DOI:10.7598/cst2016.1193

60. Kulkarni Y D, Srivastava S, Abdi S H R and Athar M, Synth React Inorg Met Org Chem., 1985, 15(8), 1043-1059; DOI:10.1080/00945718508060634

61. Khera B, Sharma A K and Kaushik N K, Polyhedron, 1983, 2, 1177-1180; DOI:10.1016/S0277-5387(00)84353-3

62. Joshi K R, Rojivadiya A J and Pandya J H, Int J Inorg Chem., 2014, 10, 1155. 